\section{Methylenetetrahydrofolate reductase homozygous mutation in a young boy with cerebellar infarction}

\author{
Alberto Spalice, ' Francesca Del Balzo, ${ }^{1}$ \\ Francesco Massimo Perla, ${ }^{2}$ \\ Enrico Properzi, ${ }^{1}$ Carla Carducci, ${ }^{3}$ \\ Italo Antonozzi, ${ }^{3}$ Paola lannetti ${ }^{3}$ \\ 'Division of Child Neurology, ${ }^{2}$ Pediatric \\ Hematology Division Department of \\ Pediatrics, and ${ }^{3}$ Department of \\ Experimental Medicine, University \\ “La Sapienza”, Rome, Italy
}

\section{Abstract}

Posterior circulation vascular occlusive disease in children is a rare and uncommonly reported event. Among the numerous risk factors, the methylenetetrahydrofolate reductase (MTHFR) mutation is considered to be a common genetic cause of thrombosis in adults and children. Recently, a link between the MTHFR mutation and cerebrovascular disorders was reported in children. Diffusion tensor imaging (DTI) is a great improvement on magnetic resonance imaging (MRI), making the in vivo anatomical and pathological study of the brain and its fibers possible. In our patient cerebellar infarction was associated with MTHFR mutation and, in a standard neurological examination, DTI revealed normal white matter tracts.

\section{Introduction}

Posterior circulation vascular occlusive disease is rare in children whereas in adults underlying vascular disease accounts for a high percentage of posterior circulation infarction. The most commonly reported cause of vertebrobasilar occlusion in children is traumatic injury to the cervical vertebral artery. ${ }^{1}$ Recently, a link between the methylenetetrahydrofolate reductase (MTHFR) mutation, which is considered to be a common genetic cause of thrombosis in adults and children, and cerebrovascular disorders was reported in children. ${ }^{2}$ The observation that most children with the MTHFR mutation do not present with cerebrovascular disorders may be a result of the requirement for additional thrombogenic risk factors. ${ }^{3}$ However, the concurrent effects of other circumstantial or genetic thrombogenic risk factors have not been established clearly, and further studies are needed to determine which associated exogenous and endogenous risk factors predispose infants with the MTHFR mutation to cerebrovascular disorders.
We report on a patient in whom cerebellar infarction was associated with MTHFR homozygous mutation.

\section{Case Report}

Our patient, a boy, was born at 36 weeks' gestation by normal vaginal delivery and had Apgar scores of 9 and 10 after 1 and 5 minutes. The family history was positive for vascular disorders: two relatives died from heart attacks. Neurological developmental milestones were normal according to age. At the age of seven years the child was hospitalized for head trauma with consequent vertigo and vomiting. No changes were observed on neuroradiological investigation and the neurological examination was normal. At the age of 13 years he had an acute episode of headache associated with vomiting. For these reasons he was referred to our Pediatric Emergency Department where he presented with headache, further vomiting, and marked vertigo. 0 n neurological examination he showed no meningeal signs, no deficit of the cranial nerves, a positive Romberg's sign, difficulty in performing coordination tests, and marked ataxia. In addition, nystagmus was present bilaterally. A CT scan was performed and demonstrated a marked hypodense lesion in the left cerebellar hemisphere. Magnetic resonance imaging (MRI) showed four areas of hyperintensity signal in T2 and Flair views without contrast enhancement. These areas where localized in the left cerebellar hemisphere, inferior part of the vermis, and right cerebellar hemisphere, respectively. Furthermore, slight compression laterally on the fourth ventricle was observed. This picture was compatible with multiple cerebellar ischemic areas (Figure 1).

The boy was hospitalized and rapidly started on anticoagulant therapy. High molecular weight heparin was administered according to the following schedule: $75 \mathrm{U} / \mathrm{kg}$ given intravenously over ten minutes, followed by a maintenance dose of $18 \mathrm{U} / \mathrm{kg} / \mathrm{h}$ for ten days. Then the child was treated with oral anticoagulants (dicoumarol), monitoring coagulation parameters daily, and maintaining an INR index of between 2 and 3 . The anticoagulation regimen was administered over two months, then dicoumarol was substituted with aspirin (100 $\mathrm{mg} /$ day). Furthermore, since the boy's diet was poor in vitamins, a multivitamin was given with the antiplatelet agent, notwithstanding the incompletely established role of vitamins in preventing endothelial damage. Over the next few days extensive coagulation studies were performed, including a complete blood count. Prothrombin time, activated partial thromboplastin time, concentrations of total and free protein $\mathrm{S}$, and antithrombin III were
Correspondence: Alberto Spalice, Second Chair of Paediatrics, Division of Child Neurology, Department of Paediatrics, University "La Sapienza", Viale Regina Elena 32400161 Roma, Italy. E-mail: a.spalice@tiscali.it

Key words: MTHFR homozygous, cerebellar infarction, children.

Received for publication: 23 June 2009.

Revision received: 1 October 2009.

Accepted for publication: 1 October 2009.

This work is licensed under a Creative Commons Attribution 3.0 License (by-nc 3.0)

(C) Copyright A. Spalice et al., 2009

Licensee PAGEPress, Italy

Pediatric Reports 2009; 1:e4

doi:10.4081/pr.2009.e4

normal; protein $\mathrm{C}$ was low (70\%; normal range 70-140\%). In addition, serum cholesterol, triglycerides, high-density lipoprotein, lowdensity lipoprotein, homocysteine, and complement components $\mathrm{C} 3$ and $\mathrm{C} 4$ concentrations were normal. Lupus anticoagulant tests and serum titers of anticardiolipin IgG and IgM, $\beta 2$ glycoprotein 1 IgG and IgM, and antinuclear and anti-DNA antibodies were normal. An evaluation for the prothrombin G20210A gene mutation was negative. The patient was found to be heterozygous for the factor $\mathrm{V}$ Leiden mutation and homozygous for the 677TT mutation of the MTHFR gene by polymerase chain reaction analysis; factor VII was normal. Moreover, the father was found to be homozygous for the MTHFR mutation and the mother was heterozygous for the MTHFR mutation.

A cardiac evaluation including electrocardiography and echocardiography was normal, and no other cause for the cerebral infarction in the child was found. A second MRI together with diffusion tensor imaging (DTI) confirmed the previous findings of cerebellar damage of recent origin. MRI of the neck showed changes at the level of the left external carotid artery and left vertebral artery. Over the next few days the clinical condition of the boy improved markedly. MRI performed one month after the event was stable. The boy was discharged from the Pediatric Department in a good neurological condition; no clinical signs of neurological deficit were observed. A follow-up MRI remained stable; no other episodes of stroke were observed. During the two years and six months of follow-up, MRI plus DTI revealed a stable neuroradiological picture of the lesion previously observed and no anomalies of the major white matter tracts, such as corpus callosum, superior longitudinal fasciculus, and corticospinal tracts. At present the boy is in good clinical health. 

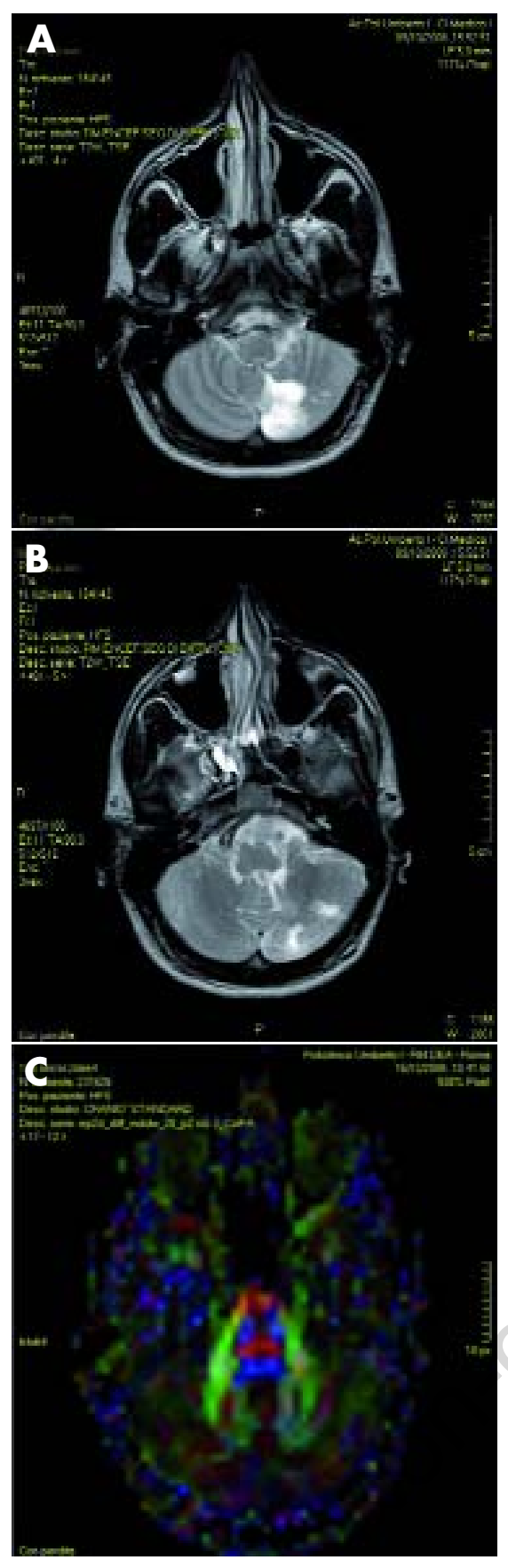

Figure 1. (A) and (B) T2-weighted axial views showing four areas of hyperintensity signal without contrast enhancement. These areas are localized in the left cerebellar hemisphere, inferior part of the vermis, and right cerebellar hemisphere. Note the slight compression laterally on the fourth ventricle. (C) Diffusion tensor imaging with fiber tracking, showing no anomalies of the major white matter tracts; corpus callosum (red), superior longitudinal fasciculus (blue), and corticospinal tracts (green).

\section{Discussion}

The clinical presentation of cerebellar infarction is diverse, and can resemble many other disorders. The main symptoms of dizziness, nausea and vomiting, gait instability, and headache are nonspecific, and are caused usually by more common and benign disorders. The important components of the neurological examination that help to identify cerebellar stroke: coordination, gait, and eye movements, are commonly omitted or abridged in primary care, particularly when symptoms might not suggest a central nervous system cause. Headache is more common in strokes in the posterior circulation than in the anterior circulation, and particularly in cerebellar infarction with nearly $40 \%$ of patients with cerebellar infarction presenting with a headache. Notably, in our patient the main clinical symptom was a posterior thunderclap headache. Although the superior cerebellar artery is the largest branch of the vertebrobasilar tree, a superior cerebellar artery territory infarction is much less common than the posterior inferior cerebellar artery syndrome of Wallenberg. ${ }^{4}$ Isolated cerebellar artery infarctions have been reported in adults, ${ }^{4}$ but cerebellar infarction is a rare event in children and the young adult. The mechanism of such infarctions may be an artery-to-artery embolism from the vertebrobasilar arteries, a cardiac source of emboli (patent foramen ovale), dissection, or fibromuscular dysplasia of the superior cerebellar artery. ${ }^{5}$ Among the risk factors for cerebral stroke, the MTHFR mutation might lead to elevation of the plasma concentrations of homocysteine, which has been associated with an increased risk of ischemic stroke. .,6-7 $^{2.0}$ However, this mutation might exert its effect via a mechanism other than elevating homocysteine concentrations. Factor V Leiden represents the most common mutation of Factor $\mathrm{V}$. It is a result of a point mutation at nucleotide 1691 of the $F V$ gene, inducing an Arg-506Glu substitution in the FV protein. The consequence of this mutation is the loss of one of three sites of cleavage for activated protein $\mathrm{C}$ (aPC) in the FV molecule, so when aPC is added to the plasma from patients with Leiden, it resists the anticoagulant effect of aPC on thrombotin production. This phenomenon is known as aPC resistance (aPCR). It has been reported that there is a prevalence for FV Leiden of $20-60 \%$ in white persons with thrombosis. The heterozygous state increases the risk for thrombosis up to $10 \%$, while the homozygous condition increases the risk 50,100-fold. ${ }^{8}$ While FV Leiden represents a well-demonstrated risk for thrombosis, the question about the role of the C677T polymorphism of the MTHFR gene in thromboem- bolism includes some aspects that need to be discussed. First, although the relationship between the increased homocysteine plasma level and cardiovascular diseases is well-documented, the molecular mechanism of endothelial damage still is not completely understood. Second, the $677 \mathrm{C}>\mathrm{T}$ polymorphism in the homozygous as well as the heterozygous state is not associated always with an increased Hct level. Meta-analyses have supported these observations. For example, Kelly and co-workers ${ }^{9}$ reported a $21 \%$ greater mean value for homocysteine plasma levels in patients with stroke, but the homozygous state of the mutation (MTHFR 677TT genotype) did not show statistical significance. ${ }^{9}$ We found the homozygous mutation of the MTHFR gene originating from the father can be considered the only risk factor for vascular accidents. The changes at the level of the left external carotid artery may be considered a normal anatomical variant and not a potential risk factor.

DTI is a great improvement on MRI in brain investigations, enabling the in vivo study of the anatomical and pathological structures of the brain and fiber tracts. Compared with routine MRI, DTI has many advantages. First, it finds the focal site of ultra-acute cerebral infarction that is displayed poorly by routine MRI; second, it clearly reveals the interruption of white matter fibers; third, it has a higher rate of spatial resolution and fewer artifacts; fourth, DTI-generated color tensor images reveal the relationship between the region of cerebral ischemia and the damaged fibers, and the three-dimensional fibrous reconstruction clearly presents the condition of the corticospinal tracts. ${ }^{10}$ In our patient, DTI demonstrated the normality of the white matter tracts. This finding is in accordance with the normal neurological condition of the boy and the absence of neurological consequences of the cerebellar stroke. As previously reported, structural abnormalities of white matter fibers are the dominant pathological change after cerebral ischemia and infarction. Such pathological changes in the microstructure of brain tissue may lead to severe ischemic stroke. In recent years, DTI has emerged as a noninvasive method to study the structure of brain white matter fibers in vivo and is promising in the evaluation of ischemic brain injury. ${ }^{10}$

In conclusion, when there are no other causes for cerebellar infarction, MTHFR mutation must be investigated. For example, when a pediatric patient presents with acute headache, a screening test for a genetic cause of thrombosis should be performed, together with MRI with DTI where possible. MRI should be preferred to a CT scan in the pediatric agegroup. Moreover, DTI seems to be the neuroradiological investigation of choice to evaluate the clinical and neuroradiological follow-up. 


\section{References}

1. Cheon JE, Kim IO, Kim WS, et al. MR diagnosis of cerebellar infarction due to vertebral artery dissection in children. Pediatr Radiol 2001;31:163-6.

2. Alluri RV, Mohan V, Komandur S, et al. MTHFR C677T gene mutation as a risk factor for arterial stroke: a hospital based study. Eur J Neurol 2005;12:1011-20.

3. Del Balzo F, Spalice A, Perla M, et al. MTHFR homozygous mutation and additional risk factors for cerebral infarction in a large Italian family. Pediatr Neurol 2008; 40:63-7.
4. Edlow JA, Newman-Toker DE, Savitz SI. Diagnosis and initial management of cerebellar infarction. Lancet Neurol 2008;7: 951-64.

5. Lin JJ, Lin KL, Chou ML, et al. Cerebellar infarction in the territory of the superior cerebellar artery in children. Pediatr Neurol 2007;37:435-7.

6. Nowak-Göttl U, Sträter R, Heinecke A, et al. Lipoprotein (a) and genetic polymorphisms of clotting factor V, prothrombin, and methylenetetrahydrofolate reductase are risk factors of spontaneous ischemic stroke in childhood. Blood 1999;94:367882.

7. Haywood S, Liesner R, Pindora S, et al.
Thrombophilia and first arterial ischaemic stroke: a systematic review. Stroke 2005; 90:402-5.

8. Montiel-Manzano G, de la Pena-Diaz A, Majluf-Cruz A, et al. National evaluation of the diagnosis of activated protein $\mathrm{C}$ resistance. Rev Invest Clin 2003;55:358-69.

9. Kelly PJ, Rosand J, Kistler JP, et al. Homocysteine, MTHFR 677C-->T polymorphism, and risk of ischemic stroke: results of a meta-analysis. Neurology 2002;59:52936.

10. Chen Z, Ni P, Zhang J, et al. Evaluating ischemic stroke with diffusion tensor imaging. Neurol Res 2008;30:720-6. 